\title{
Propuesta de un indicador para medir el comportamiento del desarrollo disciplinar de las Ciencias Bibliotecológica y de la Información en instituciones académicas
}

\author{
Salvador Gorbea Portal *
}

Ma. Maricela Piña Pozas **

Artículo recibido: 28 de enero de 2013.

Artículo aceptado: 12 de abril de 2013.

\section{RESUMEN}

Se fundamenta la propuesta de un Número Índice para medir el desarrollo disciplinar de las Ciencias Bibliotecológica y de la Información en instituciones académicas, mediante la correlación existente entre un conjunto de indicadores de potencialidades o de entrada (input) y bibliométricos o de salida (output). Para la obtención de estos indicadores se parte de un estudio sobre las potencialidades en investigación y docencia en una muestra de instituciones que realizan este tipo de actividades.

Palabras clave: Indicadores científicos; Indicadores Bibliométricos; Números Índice; Desarrollo Disciplinar; Evaluación de Instituciones.

* Instituto de Investigaciones Bibliotecológicas y de la Información, UNAM, México. portal@unam.mx

** Instituto Nacional de Salud Pública, México. maricela.pozas@insp.mx 
Proposal of an indicator to measure the performance of the disciplinary development of Library and Information Science in Academic Institutions

Salvador Gorbea-Portal and Ma--Maricela PiñaPozas

The proposal is focused on developing an index to measure the disciplinary development of Library and Information Science in academic institutions. This index proposes a correlation between a set of potential input and bibliometric output indicators. To obtain indicators of this kind, researchers carried out a study of the research and teaching potentials in a sample of institutions performing these tasks.

Keywords: Scientific Indicators; Bibliometric Indicators; Index Numbers; Discipline Development; Assessment Institutions.

\section{INTRODUCCIÓN}

T os indicadores bibliométricos con frecuencia han sido utilizados para Lundamentar el nivel de desarrollo científico alcanzado por determinada disciplina, institución o país, práctica que ha propiciado las propuestas de políticas científicas y de información sustentadas en el reconocimiento de altos niveles de productividad, impacto, visibilidad y crecimiento de la literatura científica generada en estas instancias. Esto ha acarreado consigo que siempre aparezcan mejor representados por este tipo de indicadores aquellas disciplinas, fuentes de información, instituciones y países que se encuentran en la denominada corriente principal o main stream (por su denominación en inglés) y cuyo grupo lo integran, en lo fundamental, los países de "centro" o de economías desarrolladas.

El comportamiento anterior ha suscitado controversia sobre la validez y utilidad de este tipo de indicadores en la evaluación de la ciencia, motivo por el cual el estudio, reconocimiento y definición de los indicadores bibliométricos en la literatura especializada es extenso. Éstos han sido definidos como 
parámetros que se usan para determinar y proporcionar datos sobre el comportamiento de [aspectos o regularidades] de la literatura científica (Sancho, 1990); valorados como un método objetivo, sencillo y económico para obtener información cuantitativa de las actividades de I\&D (Investigación y Desarrollo) (Cozzens, 1990) constituyen medidas obtenidas a partir del análisis estadístico de los rasgos cuantificables de la literatura científica (MaltrásBarba, 2003), son reconocidos como una de las herramienta más utilizadas para la medición del producto de la investigación científica (Russell-Barnard, 2004) y deben ser incluidos como parte de la evaluación de los resultados de la investigación publicada (Van Raan, 1996). Al mismo tiempo otros autores han proporcionado condiciones para su uso y mostrado sus limitaciones (López-Piñero y Terrada, 1992) y en ocasiones ha sido cuestionada su falta de validez cuando son utilizados de forma aislada y simplista en la evaluación y, más aún, en el caso de la ciencia, donde resulta necesario [por la complejidad de este fenómeno] utilizar varios indicadores a la vez (Sancho, 1996), además de considerarse que su aplicación debe ser complementada con la experiencia de los expertos (Gómez-Caridad y Bordons-Gangas, 1996).

En la actualidad, no obstante las continuas revisiones y críticas que han recibido, los indicadores bibliométricos han cobrado gran notoriedad con el surgimiento de los rankings (jerarquías) de universidades, fuentes y recursos de información, así como con las crecientes exigencias para la evaluación del trabajo académico y en particular de los recursos humanos dedicados a actividades de docencia, investigación y desarrollo. Estos nuevos requerimientos han llegado hasta la necesidad de institucionalizar las actividades bibliométricas en universidades españolas, tal es el caso de la creación de Unidades de Bibliometría en las de Navarra y Granada (Torres-Salinas y Jiménez-Contreras, 2012). Aunque estos autores reconocen que esta práctica bibliométrica tiene sus orígenes en los trabajos de Francis Narin en los años sesenta [y setenta], que dieron origen a la Bibliometría Evaluativa definida por el propio Narin como "el empleo de las técnicas bibliométricas, especialmente el análisis de las publicaciones y las citas, en la evaluación de la actividad científica” (Narin, 1976:335).

Sin embargo la propuesta de una nueva forma de medir y conceptualizar el desarrollo del conocimiento alcanzado en determinada disciplina en países de economía y ciencia periféricas, en los que dicha medición no dependa sólo de las aportaciones que determinado país, región o institución haga de una disciplina científica, sino de la medida en la que se correlacionen los resultados obtenidos con las potencialidades que para ello cuentan, pudiera estar aportando nuevos elementos que coadyuven a una mejor interpretación y comprensión de los indicadores bibliométricos, y por consiguiente del estado 
que guardan las regularidades presentes en el comportamiento de la producción y comunicación científicas en estos países y regiones.

La propuesta anterior sirvió de sustento teórico-metodológico en un proyecto de investigación ya concluido sobre esta problemática, en el que se elaboró un diagnóstico que identificaba los principales rasgos característicos sobre el comportamiento de las potencialidades de investigación y docencia, en una muestra de países de la región iberoamericana en los que se imparte algún tipo de docencia o se realizan investigaciones en Ciencias Bibliotecológica y de la Información. Esto con el propósito de correlacionar a posteriori estos resultados con los obtenidos de carácter bibliométrico, y determinar con ello la tendencia del desarrollo que estos países alcanzan en función de las aportaciones que realizan a estas disciplinas, según los recursos y potencialidades de que disponen (Gorbea-Portal, 2010).

Otra aportación del proyecto antes referido se presentó en una tesis de doctorado en la que se correlacionaron indicadores de potencialidades y bibliométricos para medir el desarrollo disciplinar en las Ciencias Bibliotecológica y de la Información en México, mediante el empleo de un Número Índice (Piña-Pozas, 2011). Estos dos resultados de investigación sentaron las bases para el desarrollo de un nuevo proyecto relacionado con el comportamiento métrico del desarrollo disciplinar iberoamericano en las Ciencias Bibliotecológica y de la Información, para lo cual fue necesario el perfeccionamiento y rediseño de los cuestionarios utilizados para identificar los indicadores de potencialidades y la ampliación de variables e indicadores, a partir de los cuales se rediseñó el Número Índice utilizado en la tesis anterior.

Por eso el presente artículo tiene como objetivo presentar una nueva propuesta de indicador que mida el desarrollo disciplinar y muestre la metodología empleada en la obtención de los resultados que se esperan lograr en el actual proyecto de investigación, orientado hacia la medición del comportamiento métrico del desarrollo disciplinar en Ciencias Bibliotecológica y de la Información en los países iberoamericanos.

\section{Material y MÉTOdo}

\section{Fuente}

Para desarrollar esta segunda investigación se tomó como fuente principal, al igual que en el proyecto anterior, un sistema de cuestionarios dirigido a la recolección de los datos sobre potencialidades de investigación y docencia, así como los referidos a la producción científica generada por las propias instituciones 
encuestadas. Este sistema de cuestionarios en la actualidad se aplica a instituciones que cuentan con programas académicos o con proyectos de investigación relacionados con estas disciplinas, de las cuales y a modo de ejemplo se seleccionaron cinco para comprobar el índice propuesto en este trabajo.

El sistema de cuestionarios diseñado para el proyecto anterior, y rediseñado para el actual, consta de tres tipos de cuestionario:

1. cuestionario institucional,

2. cuestionario de recursos humanos y

3. cuestionario de proyectos de investigación.

En los cuestionaros aplicados en las instituciones seleccionadas para este trabajo se solicitó cubrir el periodo de 2007 a 2011. Aunque las preguntas estuvieron diseñadas para ser respondidas durante ese lapso, los cuestionarios incluían preguntas de planeación, como es el caso de la pregunta 11 del cuestionario institucional que se refiere a la planeación de futuras actividades para otro periodo.

\section{Variables, indicadores y procesamiento de los datos}

Para definir el conjunto de variables e indicadores representativos de este trabajo de investigación, se tomaron en cuenta experiencias previas de autores que han estudiado y propuesto indicadores relacionados con el tipo de indicador que aquí se presenta (Sancho, 1990; OCDE, 1993, 1995; Suárez-Balseiro, 2004), una relación de las variables seleccionadas se muestra en la Tabla 1.

Tabla 1. Relación de variables seleccionadas, según valores

\begin{tabular}{|c|c|}
\hline Variables de Potencialidades & Variables Bibliométricas \\
\hline Recursos Humanos & Producción Científica \\
\hline Cargo: & Tipología Documental: \\
\hline$\cdot$ Docentes & $\cdot$ Artículos \\
\hline$\cdot$ Investigadores & $\cdot$ Libros \\
\hline Grado: & $\cdot$ Capítulos de libros \\
\hline$\cdot$ Doctorado & $\cdot$ Tesis \\
\hline$\cdot$ Maestría & Lugar de Publicación: \\
\hline$\cdot$ Licenciatura & $\cdot$ Nacional \\
\hline Proyectos de Investigación: & $\cdot$ Extranjero \\
\hline$\cdot$ En Curso & Fuente de Indización: \\
\hline$\cdot$ Terminados & $\cdot$ Base de Datos Especializada \\
\hline Programas Docentes: & $\cdot$ Índice de Citas \\
\hline$\cdot$ Licenciatura & Idioma de la Publicación: \\
\hline$\cdot$ Maestría &
\end{tabular}




\begin{tabular}{|l|c|}
\hline$\cdot$ Doctorado & $\cdot$ Español \\
\hline$\cdot$ Otro & $\cdot$ Inglés \\
\hline Matrícula de Alumno: & $\cdot$ Portugués \\
\hline$\cdot$ Licenciatura & $\cdot$ Francés \\
\hline$\cdot$ Maestría & Origen de publicación de las Revistas Fuente: \\
\hline$\cdot$ Doctorado & $\cdot$ Nacional \\
\hline$\cdot$ Otro & $\cdot$ Extranjera \\
\hline Año & Año \\
\hline Identificador de la Institución & Identificador de la Institución \\
\hline
\end{tabular}

Con las variables anteriores se construyeron las matrices de datos originales que se utilizaron en esta investigación, estas variables se obtienen con el sistema de cuestionarios aplicados a cada institución y muestran el comportamiento que éstas asumen de acuerdo con los valores que representan durante el periodo estudiado, y a partir de las cuales se construye el sistema de indicadores que se relacionan en la Tabla 2.

Tabla 2. Relación de indicadores seleccionados según tipo para cada Institución

\begin{tabular}{|c|c|}
\hline Indicadores de Potencialidades & Indicadores Bibliométricos \\
\hline $\begin{array}{l}\text { - Recursos Humanos por año, según cargo que se } \\
\text { ocupe }\end{array}$ & $\begin{array}{l}\text { - Producción científica por año, según tipología } \\
\text { documental }\end{array}$ \\
\hline $\begin{array}{l}\text { - Recursos Humanos por año, según el último grado } \\
\text { obtenido }\end{array}$ & $\begin{array}{l}\text { - Producción científica por año, según lugar de } \\
\text { publicación }\end{array}$ \\
\hline - Proyectos de Investigación por año, según tipología & $\begin{array}{l}\text { - Artículos científicos por año, según fuente de } \\
\text { indización }\end{array}$ \\
\hline $\begin{array}{l}\text { - Programas docentes por año, según el nivel } \\
\text { académico impartido }\end{array}$ & $\begin{array}{l}\text { - Producción científica por año, según idioma de } \\
\text { publicación }\end{array}$ \\
\hline $\begin{array}{l}\text { - Matrícula de Alumnos por año, según el nivel } \\
\text { cursado }\end{array}$ & $\begin{array}{l}\text { - Artículos científicos por año, según el origen de } \\
\text { la revista }\end{array}$ \\
\hline $\begin{array}{l}\text { - Valor Promedio de Recursos Humanos según el } \\
\text { cargo que ocupan }\end{array}$ & $\begin{array}{l}\text { - Valor Promedio de la Producción científica según la } \\
\text { tipología documental }\end{array}$ \\
\hline $\begin{array}{l}\text { - Valor Promedio de los Recursos Humanos, según el } \\
\text { último grado obtenido }\end{array}$ & $\begin{array}{l}\text { - Valor Promedio de la Producción científica según el } \\
\text { lugar de publicación }\end{array}$ \\
\hline $\begin{array}{l}\text { - Valor Promedio de Proyectos de Investigación } \\
\text { según su tipología }\end{array}$ & $\begin{array}{l}\text { - Valor Promedio de artículos científicos según su } \\
\text { fuente de indización }\end{array}$ \\
\hline $\begin{array}{l}\text { - Valor Promedio de Programas docentes según el } \\
\text { nivel académico impartido }\end{array}$ & $\begin{array}{l}\text { - Valor Promedio de la Producción científica según el } \\
\text { idioma de publicación }\end{array}$ \\
\hline $\begin{array}{l}\text { - Valor Promedio de Matrícula de Alumnos por año } \\
\text { según el nivel cursado }\end{array}$ & $\begin{array}{l}\text { - Valor Promedio de artículos científicos según el } \\
\text { origen de la revista }\end{array}$ \\
\hline $\begin{array}{l}\text { - Estructura porcentual de Recursos Humanos según } \\
\text { el cargo que ocupan }\end{array}$ & $\begin{array}{l}\text { - Estructura porcentual de la Producción científica } \\
\text { según la tipología documental }\end{array}$ \\
\hline $\begin{array}{l}\text { - Estructura porcentual de Recursos Humanos según } \\
\text { el último grado obtenido }\end{array}$ & $\begin{array}{l}\text { - Estructura porcentual de la Producción científica } \\
\text { según el lugar de publicación }\end{array}$ \\
\hline $\begin{array}{l}\text { - Estructura porcentual de Proyectos de Investiga- } \\
\text { ción según la tipología }\end{array}$ & $\begin{array}{l}\text { - Estructura porcentual de artículos científicos } \\
\text { según la fuente de indización }\end{array}$ \\
\hline
\end{tabular}




\begin{tabular}{|c|c|}
\hline $\begin{array}{l}\text { - Estructura porcentual de Programas docentes } \\
\text { según el nivel académico impartido }\end{array}$ & $\begin{array}{l}\text { - Estructura porcentual de la Producción científica } \\
\text { según el idioma de publicación }\end{array}$ \\
\hline $\begin{array}{l}\text { - Estructura porcentual de Matrícula de Alumnos por } \\
\text { año según el nivel cursado }\end{array}$ & $\begin{array}{l}\text { - Estructura porcentual de artículos científicos según } \\
\text { el origen de la revista }\end{array}$ \\
\hline $\begin{array}{l}\text { - Proyección de Crecimiento de Recursos Humanos } \\
\text { según el cargo que ocuparon para el } 2012\end{array}$ & $\begin{array}{l}\text { - Proyección de Crecimiento de la Producción } \\
\text { científica según la tipología documental }\end{array}$ \\
\hline $\begin{array}{l}\text { - Proyección de Crecimiento de Recursos Humanos } \\
\text { según el último grado obtenido para el } 2012\end{array}$ & $\begin{array}{l}\text { - Proyección de Crecimiento de la Producción } \\
\text { científica según el lugar de publicación }\end{array}$ \\
\hline $\begin{array}{l}\text { - Proyección de Crecimiento de Proyectos de Investi- } \\
\text { gación según la tipología para el } 2012\end{array}$ & $\begin{array}{l}\text { - Proyección de Crecimiento de artículos científicos } \\
\text { según la fuente de indización }\end{array}$ \\
\hline $\begin{array}{l}\text { - Proyección de Crecimiento de Programas docentes } \\
\text { según el nivel académico impartido para el } 2012\end{array}$ & $\begin{array}{l}\text { - Proyección de Crecimiento de la Producción } \\
\text { científica según el idioma de publicación }\end{array}$ \\
\hline $\begin{array}{l}\text { - Proyección de Crecimiento de Matrícula de Alum- } \\
\text { nos por año según el nivel cursado para el } 2012\end{array}$ & $\begin{array}{l}\text { - Proyección de Crecimiento de artículos científicos } \\
\text { según el origen de la revista }\end{array}$ \\
\hline
\end{tabular}

En la tabla anterior se relacionan todos los indicadores (unidimensionales) que fueron utilizados para identificar el comportamiento de regularidades, tanto en las potencialidades como en las referentes a los resultados de la investigación; es decir, los de producción científica y los obtenidos de las matrices que se muestran de forma completa en las Tablas 1 y 2 del Anexo, una muestra abreviada de estas matrices se presenta en las Figuras 1 y 2.

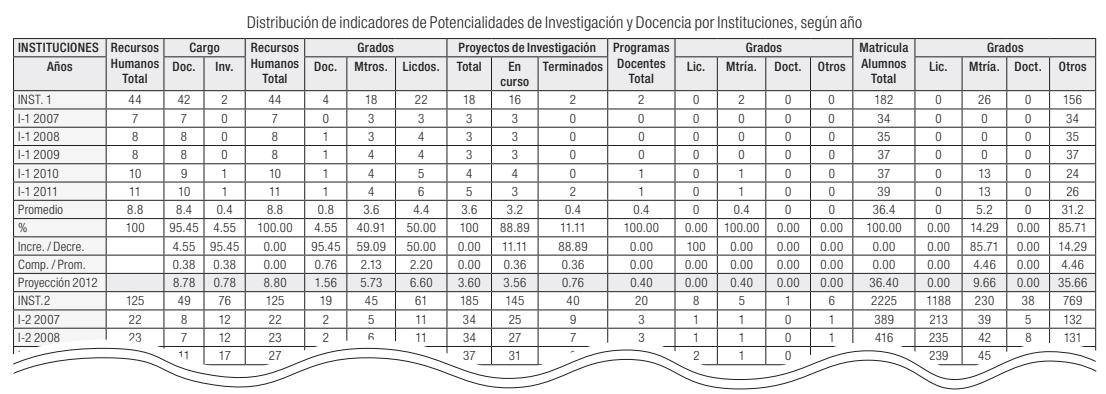

Figura 1. Matriz de Indicadores de Potencialidades en Investigación y docencia por Instituciones, según año.

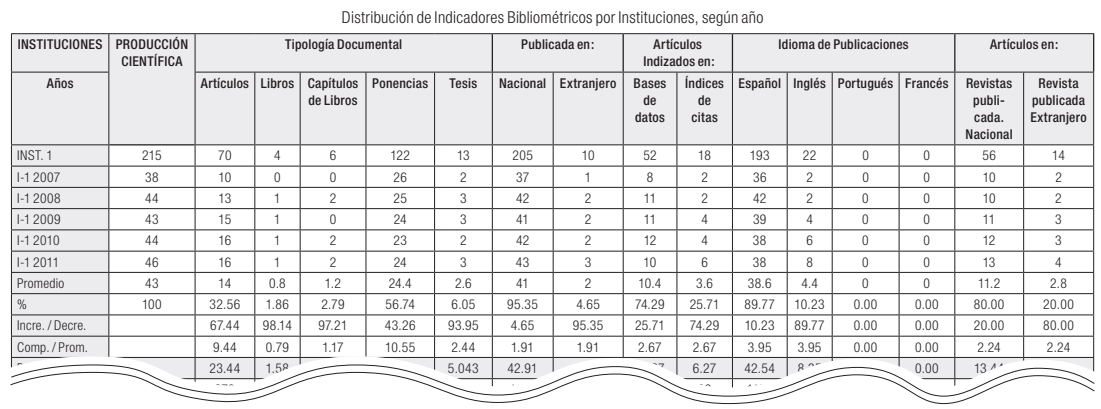

Figura 2. Matriz de Indicadores Bibliométricos por Instituciones, según año. 
En estas matrices, además de integrarse los valores absolutos de cada variable, se le han agregado también: el valor promedio y la estructura porcentual de cada variable, el diferencial del incremento o decremento del valor promedio respecto al $100 \%$ y la tasa de comprobación del promedio, estos dos últimos para obtener la proyección o expectativa de crecimiento de cada institución, según la variable para el año 2012. Todos estos cálculos se realizaron tanto para los indicadores de potencialidades como para los bibliométricos.

El procedimiento utilizado para el cálculo del número base o crecimiento esperado para cada variable se obtiene a partir de la sumatoria de los valores obtenidos de cada una de ellas en los años seleccionados, y a partir del total que resulta de cada variable se calcula su valor promedio; después se halla su estructura porcentual correspondiente a cada columna, es decir, a cada promedio le corresponde un porcentaje de acuerdo con el total general de cada variable. Utilizando como ejemplo los valores obtenidos en la variable "artículos" correspondiente a la matriz de indicadores bibliométricos se obtienen los valores mostrados en la Tabla 3.

Tabla 3. Procedimiento empleado en el cálculo de la expectativa de crecimiento de las variables seleccionadas

\begin{tabular}{|l|l|}
\hline \multicolumn{1}{|c|}{ Celda en la Tabla } & \multicolumn{1}{c|}{ Descripción / Fórmula } \\
\hline Promedio & $\sum$ (Artículos) / Núm. Años =70/5=14 \\
\hline$\%$ & Promedio de la $\sum$ total = 43 (100\%), entonces (14) (100)/43=32.55 \\
\hline Incremento / Decremento & $\begin{array}{l}\text { Diferencial del Incremento o Decremento del valor promedio respecto al 100\% } \\
\text { Para saber cuánto creció o decreció la variable se le resta al 100\% el porcentaje } \\
\text { que ésta representa del total (100 - 32.55) =67.44 }\end{array}$ \\
\hline Comprobación / Promedio & $\begin{array}{l}\text { Tasa de Comprobación del Promedio: el resultado anterior se multiplica por el } \\
\text { promedio y se divide entre 100 (67.44)(14) / 100 = 9.44 }\end{array}$ \\
\hline Proyección 2012 & $\begin{array}{l}\text { Expectativa de crecimiento de cada institución, según variable para el año } 2012 . \\
\text { Finalmente, el valor anterior se suma al promedio y se obtiene la proyección de } \\
\text { la variable para el 2012, entonces 9.44 + 14 = 23.4 }\end{array}$ \\
\hline
\end{tabular}

Los valores obtenidos en el cálculo de la proyección de cada variable para el año 2012 (23.4 en el caso de los artículos en el ejemplo de la Tabla 3) indican la proporción en la que crecerá cada una de las variables, las bibliométricas o las de potencialidades, para ese año, y de forma directa determinan el ritmo de crecimiento del desarrollo de cada institución. Además, este valor calculado para cada año base, obtenido del comportamiento de los años anteriores, puede ser comparado con los valores que se obtengan en los años subsiguientes y graficado para explicar su comportamiento durante un quinquenio, una década u otro periodo que se quiera analizar.

Los Números Índice muestran el comportamiento o cambio de una variable de un periodo a otro y este valor es expresado en un tanto por ciento, es 
decir entre 0-100. Sin embargo, por lo general se omite el signo de \% y en algunos casos el valor resultante puede llegar a superar el $100 \%$. Cuando esto ocurre, autores como Mason y Douglas precisan qué hacer y con un ejemplo orientado a la actividad general de una empresa explican: "que si el valor obtenido para 1989 es de 157.1, esto se interpreta [como] que la actividad de negocios de esa empresa aumentó $57.1 \%$ desde el periodo base (seleccionando arbitrariamente como 1977) hasta 1989" (Mason y Douglas, 1992: 695-713).

Esta precisión metodológica ha sido tomada en cuenta con los valores obtenidos por encima del 100\% en ambas matrices, las cuales aparecen en el anexo de este trabajo (ver Tablas 1 y $2 \mathrm{del}$ Anexo) y donde se puede observar que aquellas celdas que rebasan el $100 \%$ han sido sombreadas para destacar esta peculiaridad; una vez reducidos estos valores como se indica se pueden observar los resultados definitivos sobre el crecimiento de las variables para el 2012 en las subsiguientes Tablas 3 y 4 del Anexo, y en los gráficos de las Figuras 4 y 5 del apartado de Resultados.

\section{Aspectos teórico-metodológicos a considerar para el Diseño de un Número Índice}

El diseño del indicador propuesto se sustenta en la teoría de los Números Índice (NI). Un NI es un indicador diseñado para describir los cambios de una variable en el tiempo; es decir, su evolución a lo largo de algún periodo determinado. Los NI intentan reflejar:

- la evolución en la cantidad de un determinado servicio, o bien en un conjunto de ellos;

- la evolución en el valor de un bien o servicio, así como en un conjunto de ellos.

La productividad se mide normalmente como un índice de cantidad de resultados (outputs) por un índice de cantidades de entrada (inputs). Los índices son necesarios debido a la heterogeneidad de los bienes y servicios, ya que no se permite simplemente la suma de unidades de diferentes tipos de productos, tal y como ocurre con las unidades de análisis de este estudio; es decir, no se pueden sumar publicaciones con proyectos, ni con la matrícula escolar, por ejemplo.

Los índices más utilizados son los de Laspeyres, de Paasche, de Fisher (una media geométrica de los índices de Laspeyres y Paasche) y de Törnqvist (una media geométrica ponderada de sus componentes) (Caves, Christensen y Diewert, 1982). 
Dado que los índices de Laspeyres y de Paasche se usan para medidas individuales de outputs e inputs (entradas y salidas) con precios o cantidades, para esta investigación se utilizó el primero, que indica los cambios en la cantidad del valor total utilizando un periodo de tiempo como base; es decir, cantidades individuales para compararlas con un periodo de tiempo base (Squires, 1988).

Cabe destacar que para fines de esta propuesta este tipo de indicadores se utiliza para medir el nivel de desarrollo disciplinar alcanzado en un campo temático o conjunto de disciplinas de un país objeto de estudio, conformado a partir del cálculo del indicador para cada una de las instituciones existentes en el país dedicadas a las actividades académicas en la disciplina o campo disciplinar objeto de estudio. En este sentido se parte del criterio de que el desarrollo disciplinar alcanzado por un país en determinado campo o disciplina puede ser considerado como un bien social del país en cuestión, por lo que la interpretación de su resultado se fundamenta mediante la correlación existente entre la salida (productos) y la entrada (potencialidades) que se obtienen en un sistema Ciencia-Investigación-Desarrollo (C\&I\&D).

Para la operatividad del Número Índice o indicador propuesto, se requiere seleccionar un periodo base, que adquiere gran importancia debido a que los resultados obtenidos tendrán un sentido conceptual respecto de él, razón por la cual este periodo debe contar con características de normalidad (Ribeiro, Rodríguez y Cervini, 2008). El periodo base seleccionado para la comprobación del indicador propuesto fue el comprendido entre 2007-2011; mientras que el año calculado en correspondencia con el periodo seleccionado fue el 2012.

Con el cálculo del NI se encuentra el cociente del valor actual entre un valor base, se multiplica el número resultante por 100 y se expresa como porcentaje. Asimismo, se encuentran los Números Índice complejos o compuestos para cuantificar las variaciones de un grupo de variables. En estos NI, se calcula uno correspondiente a cada variable y se combinan para obtener un NI complejo en resumen (Sánchez-Fernández, 2004), método seguido en el diseño del Índice de Desarrollo Disciplinar para cada institución propuesto en esta investigación.

\section{Modelo de operatividad entre las variables y los indicadores}

El modelo de operatividad resultante entre las variables y los indicadores es de tipo multidimensional y parte, como ya se indicó anteriormente, de la implementación de un sistema de cuestionarios que se trabajó en tres dimensiones: desarrollo institucional, recursos humanos y proyectos de investigación, encaminado a obtener tanto información como los indicadores sobre las potencialidades de 
investigación y docencia, modelo que se mueve en otros dos planos o dimensiones: el institucional y el nacional; esta relación se muestra en la Figura 3.

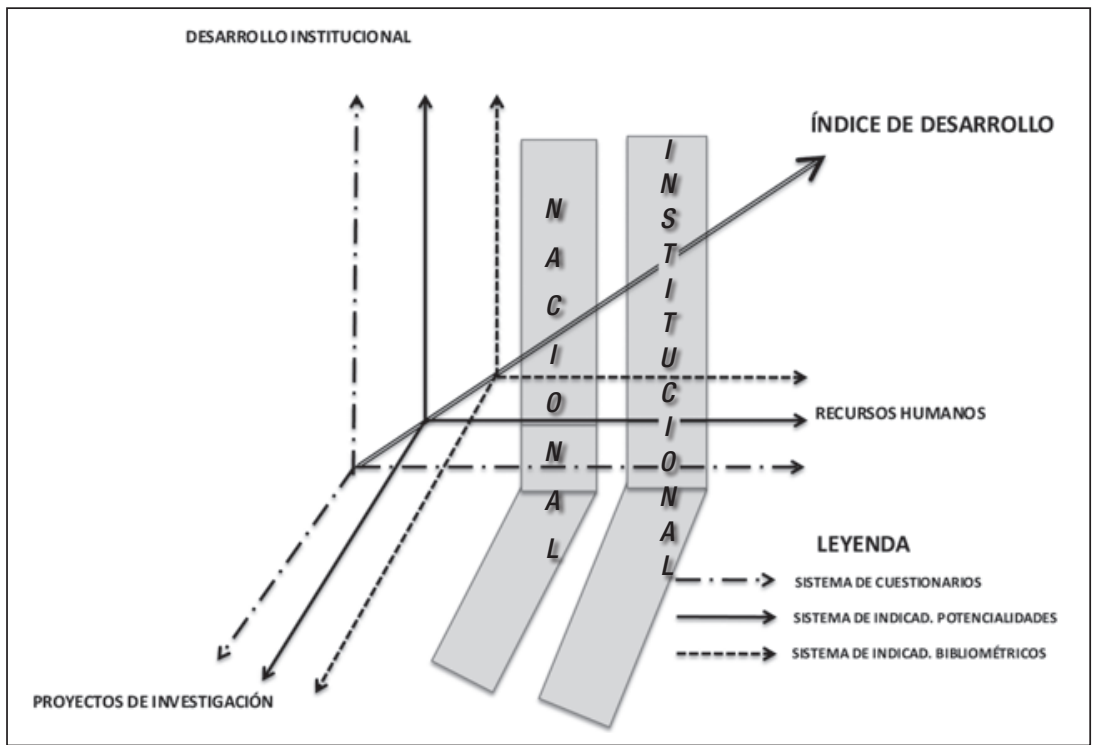

Figura 3. Modelo de operatividad de las variables y de los indicadores en la obtención del Índice de Desarrollo Disciplinar

En esta multidimensionalidad se integran al Modelo el Sistema de Indicadores sobre potencialidades y el Sistema de Indicadores Bibliométricos, los cuales también se mueven en los tres planos: institucional, recursos humanos y proyectos de investigación. Estos sistemas permitieron la elaboración de diagnósticos institucionales y nacionales en el Proyecto de Investigación anterior, y en ellos se presenta una panorámica general del comportamiento de las potencialidades de investigación y docencia en determinada institución o país, antecedentes tomados en cuenta para medir y comprobar la factibilidad del indicador propuesto y, por consiguiente, la medición del desarrollo institucional y disciplinar, objetivo principal del actual proyecto.

\section{AnÁlisis de los Resultados}

Los resultados obtenidos en esta propuesta se orientan en dos direcciones, la primera determina el grado de crecimiento en cada una de las variables que han sido seleccionadas para este estudio, tanto las de potencialidades como las bibliométricas, mientras que la segunda calcula el comportamiento del 
desarrollo disciplinar de cada una de las instituciones encuestadas, mediante la propuesta de un indicador, además de evidenciar las (dis)similitudes que se dan entre ambos resultados.

\section{Expectativas del crecimiento institucional, según variables seleccionadas}

De acuerdo con lo explicado en el apartado metodológico y con el auxilio de la teoría de los Números Índice se obtienen las tasas de crecimiento del sistema de variables para el año 2012, partiendo del análisis de los valores de estas variables en un periodo base o de comparación entre los años 2007-2011. Los resultados de estos cálculos se encuentran distribuidos en detalle por cada institución y variable en las Tablas 3 y 4 del Anexo, las cuales sirvieron de fuente para elaborar los gráficos que se presentan en las Figuras 4 y 5.

En la Figura 4, correspondiente a las variables bibliométricas que representan el comportamiento de las principales regularidades observadas en la producción científica generada en las cinco instituciones, se destacan la 2 y la 4 con una tasa de crecimiento promedio de 29.06 y 23.28 respectivamente, seguidas de la institución 5 con 21.16, mientras que las instituciones 1 y 3 presentan tasas similares de 13.53 y 14.16 cada una. Este comportamiento infiere cierta regularidad en la proporción con la que se supone que estas instituciones debieron crecer en materia de productos derivados de su actividad docente y de investigación, puesto que entre la de mayor y menor crecimiento se observa una prudente diferencia de 15.53 , comportamiento que puede ser observado en la gráfica de la Figura 4.

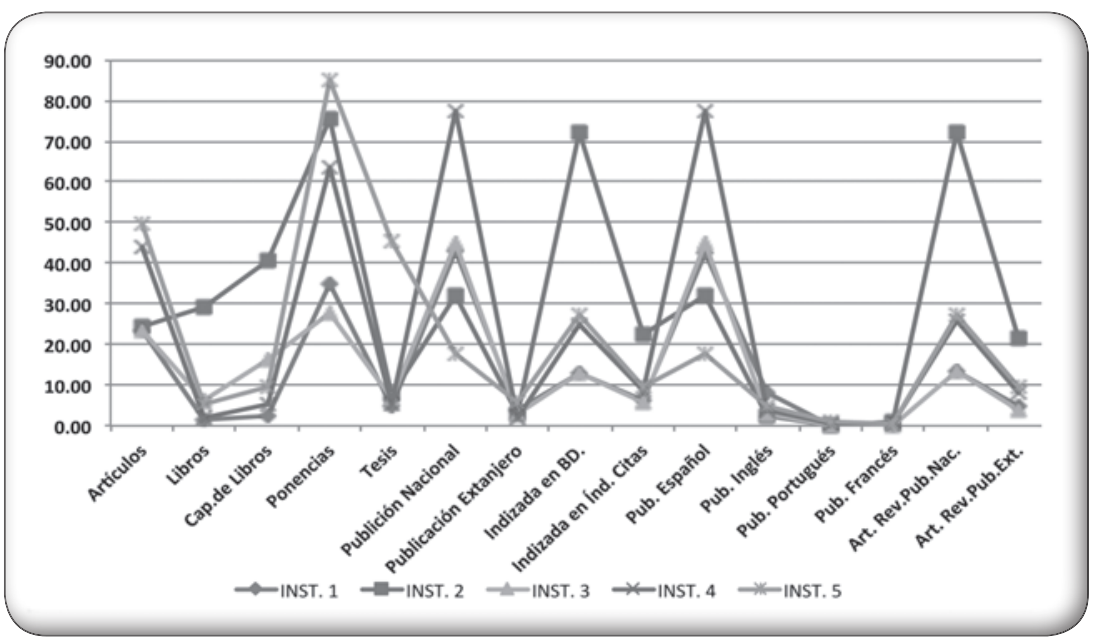

Figura 4. Expectativa de crecimiento por institución, según variables bibliométricas seleccionadas 
Las tasas de crecimiento de las variables sobre las potencialidades de que disponen estas instituciones para generar sus productos de investigación no presentan un comportamiento muy disímil a las bibliométricas; en este caso las instituciones 5 y 2 aparecen como las de mayor crecimiento promedio de sus variables de potencialidades, con tasas de 25.40 y 22.57, respectivamente, sigue después la institución 4 con una tasa de 15.95 y un poco más distantes, como en el caso anterior, las instituciones 3 y 1 con tasas de 6.27 y 5.21 . En este caso la brecha entre la institución de mayor crecimiento promedio (INST-5), respecto a las potencialidades con las que cuentan para generar sus productos científicos, y la de menor (INST-1), es más pronunciada y arroja una diferencia de 20.19, lo que indica que en materia de recursos y potencialidades estas instituciones presentan una mayor desigualdad, cosa que no ocurre, al menos en menor medida, al mostrar su crecimiento en sus productos científicos. El comportamiento gráfico de estas variables se presenta en la Figura 5 .

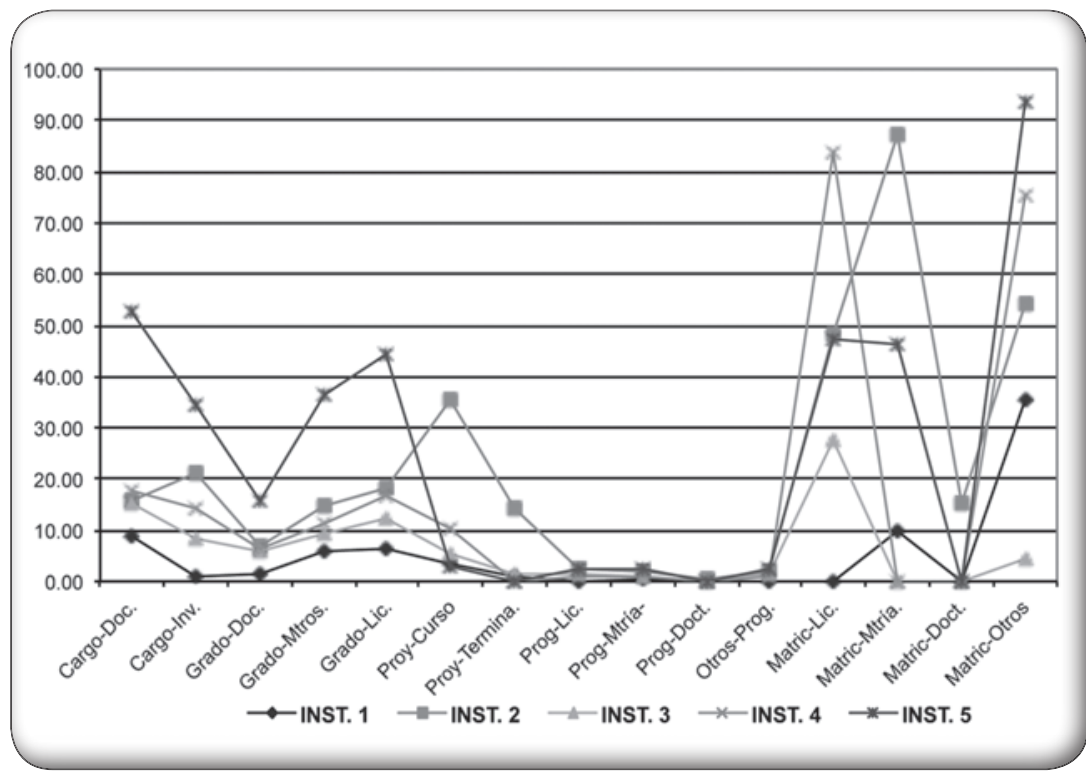

Figura 5. Expectativa de crecimiento por institución, según variables de potencialidades seleccionadas

El comportamiento identificado entre estos dos sistemas de variables e indicadores podría estar confirmando la teoría de que no siempre las instituciones que cuentan con más potencial científico (recursos humanos, matrícula escolar, grado académico, entre otros) son las que más producción generan. Es precisamente de este supuesto del que parte la propuesta de diseñar 
un indicador que, basado en la misma teoría de los Números Índice con la que se obtuvieron estas tasas de crecimiento, evidencie la presencia de un desarrollo en instituciones de economía periféricas que no se sustente, como suele ocurrir, en privilegiar altos niveles de producción sobre la base de niveles de potencialidades más altos. A continuación se presenta la propuesta de un indicador que, utilizando las mismas variables del análisis anterior, demuestra comportamientos muy diferentes a éstos.

\section{Diseño y aplicación del Índice de Desarrollo Disciplinar en instituciones académicas}

El Índice de Desarrollo Disciplinar ha sido diseñado tomando en cuenta las bases teóricas y metodológicas de los Números Índice, los cuales como ya quedó indicado, permiten medir qué tanto ha cambiado una variable con el tiempo, así como cuál ha sido su evolución a lo largo de un periodo determinado. Este índice se calcula encontrando el cociente del valor actual dividido entre un valor base, y posteriormente se multiplica el número resultante por 100, con el fin de expresar el índice como un porcentaje, que finalmente es el porcentaje o valor relativo (Sánchez-Fernández, 2004).

La construcción del Índice de Desarrollo Disciplinar se establece a partir de los indicadores de entrada (sobre potencialidades de investigación y docencia) y los de salida (los referentes a la producción y comunicación científica). Los elementos teóricos y relacionales que se indican en el modelo se insertan en un mismo marco teórico, y por eso los resultados obtenidos en este sentido amplían el marco teórico del cual parten.

La propuesta se circunscribe a medir la razón que existe entre los indicadores bibliométricos y los de potencialidades, para con ello identificar el nivel de desarrollo disciplinar alcanzado en las instituciones académicas en determinado campo de conocimiento.

El modelo matemático general de un Número Índice del cual se parte para el diseño del indicador propuesto toma como base la formulación siguiente:

$$
N i_{(t)}=\frac{\sum_{i=1}^{n}\left[\frac{x_{i j}}{y_{i j}}\right]}{N}
$$

En el que:

$\mathrm{Ni}(t)=$ Número Índice general

$\Sigma=$ sumatoria de $i=1$ hasta $n$ 
$i=$ valor inicial, llamado límite inferior

$n=$ valor final, llamado límite superior

$x i j=$ indicadores de salida $x i$ para cada $j$ institución

$y i j=$ indicadores de entrada $y i$ para cada $j$ institución

$N=$ número base

A partir del cual se desarrolla una formulación para medir el comportamiento de los indicadores de entrada o de potencialidades (input), y otra para los indicadores de salida (output) o bibliométricos.

- Índice de desarrollo institucional de indicadores bibliométricos

El índice de desarrollo institucional de indicadores bibliométricos es la sumatoria de $i=1$ hasta $n$, con un valor inicial $i$ llamado límite inferior y un valor final $n$ llamado límite superior, que al sustituir los componentes de su formulación matemática por los valores obtenidos del conjunto de indicadores, queda calculado para cada institución de la manera siguiente:

$$
I b i j=\sum_{i=1}^{n}\left[\frac{b x j}{N i}\right] \quad I b i j=\sum_{i=1}^{n}\left[\frac{b x i}{N j}\right]
$$

Donde:

$$
\begin{aligned}
I b i j= & \text { índice de indicadores bibliométricos para una insti- } \\
& \text { tución } j \text { en particular } \\
b x j= & \text { indicadores bibliométricos } x j \text { entre cada } j \text { año } \\
b x i= & \text { indicadores bibliométricos } x i \text { entre cada } i \text { indicador } \\
N j= & \text { número de años } \\
N i= & \text { número de indicadores }
\end{aligned}
$$

Sustituyendo los valores identificados en la formulación anterior se obtienen los resultados siguientes:

Institución 1

$$
I i b=\frac{215+215+70+215+70}{5}=\frac{785}{5}=157=1.57
$$

Institución 2

$$
I i b=\frac{1160+1160+370+1160+370}{5}=\frac{4220}{5}=844=8.44
$$

Institución 3

$$
I i b=\frac{224+224+69+224+69}{5}=\frac{810}{5}=162=1.62
$$


Institución 4

$$
I i b=\frac{387+387+133+387+133}{5}=\frac{1427}{5}=285=2.85
$$

Institución 5

$$
I i b=\frac{588+588+142+588+142}{5}=\frac{2052}{5}=410=4.10
$$

- Indice de desarrollo institucional de indicadores sobre potencialidades De igual forma, el índice de desarrollo institucional de indicadores de potencialidades es la sumatoria de $i=1$ hasta $n$, con un valor inicial $i$ llamado límite inferior y un valor final $n$ llamado límite superior, y su formulación matemática como en el caso anterior es la siguiente:

$$
\operatorname{Iip}=\sum_{i=1}^{n}\left[\frac{p y j}{N i}\right] \quad \text { Iip }=\sum_{i=1}^{n}\left[\frac{p y i}{N j}\right]
$$

Donde:

$$
\begin{aligned}
I i p= & \text { índice de indicadores de potencialidades por institución } \\
p y j= & \text { indicadores de potencialidades } y j \text { entre cada } j \text { año } \\
p y i= & \text { indicadores de potencialidades } y i \text { institución para ca- } \\
& \text { da } i \text { indicador } \\
N j= & \text { número de instituciones } \\
N i= & \text { número de indicadores }
\end{aligned}
$$

Sustituyendo los valores identificados en la formulación anterior se obtienen los resultados siguientes:

Institución 1

$$
I i p=\frac{44+44+18+2+182}{5}=\frac{290}{5}=57.2=0.57
$$

Institución 2

$$
I i p=\frac{125+125+185+20+2285}{5}=\frac{2680}{5}=536=5.36
$$

Institución 3

$$
I i p=\frac{84+84+28+12+780}{5}=\frac{988}{5}=197=1.97
$$

Institución 4

$$
I i p=\frac{108+108+51+9+1593}{5}=\frac{1869}{5}=373=3.73
$$

Institución 5

$$
I i p=\frac{301+301+14+21+2877}{5}=\frac{3514}{5}=702=7.02
$$


- Índice de desarrollo institucional

El índice de desarrollo institucional es la sumatoria de los indicadores bibliométricos correspondientes a cada institución participante, entre la sumatoria de los indicadores de potencialidades, y al sustituir su formulación matemática por los valores calculados en las dos formulaciones anteriores, queda resuelto para cada institución de la manera siguiente:

$$
I d i=\frac{\sum_{i=1}^{n}\left[\frac{b x i j}{p y i j}\right]}{\mathrm{N}}
$$

\section{Institución 1}

$$
I d i=\frac{1.57}{0.58}=\frac{2.70}{5}=0.54 \quad I d i=0.54
$$

Institución 2

$$
I d i=\frac{8.44}{5.36}=\frac{1.57}{5}=0.31 \quad I d i=0.31
$$

Institución 3

$$
I d i=\frac{1.62}{1.97}=\frac{0.82}{5}=0.16 \quad I d i=0.16
$$

\section{Institución 4}

$$
I d i=\frac{2.85}{3.73}=\frac{0.76}{5}=0.15 \quad I d i=0.15
$$

Institución 5

$$
I d i=\frac{4.10}{7.02}=\frac{0.58}{5}=0.11 \quad I d i=0.11
$$

- Índice de desarrollo disciplinar global para todo el país o región

De acuerdo con los resultados obtenidos en cada una de las instituciones empleadas para este estudio y dada la relevancia que éstas tienen en su país, se podría inferir el nivel de desarrollo que alcanza la disciplina en todo el país, mediante el cálculo de un valor promedio entre todas las instituciones, el cual puede ser representativo de todo el país o de una región según sea el caso. Un cálculo realizado con los resultados finales obtenidos en este sentido revela el comportamiento siguiente:

$$
I d d=\frac{0.55+0.31+0.16+0.15+0.11}{5}=\frac{1.27}{5}=0.25
$$

Este resultado indica que el Índice de Desarrollo Disciplinar promedio para todo el país se encuentra en una tasa de 0.25 , por supuesto 
respecto a las relaciones resueltas anteriormente por la razón existente entre los indicadores bibliométricos y los de potencialidades, además de concebir el desarrollo disciplinar derivado de la proporcionalidad antes explicada.

Como parte del diseño de este indicador y con el propósito de determinar los elementos de su interpretación se establece una regla de decisión en los términos que se explican a continuación.

- Regla de decisión

El resultado del Idd es un valor que se estima entre 0 y 1, de ahí que:

- Si el resultado máximo calculado del $I d d$ es < que el valor 0.5, entonces las potencialidades resultaron superiores a la obtención de resultados; es decir, la tendencia a la baja significa que hay mayores recursos que los resultados que se obtienen.

- Si el resultado máximo calculado del Idd es > que el valor 0.5, entonces las potencialidades tienen un aceptado nivel de utilización; es decir, la tendencia a la alta significa que hay mayor eficiencia en el uso de las potencialidades para la obtención de los resultados de investigación, lo cual coadyuva a un mejor nivel de desarrollo disciplinar de determinada institución, país o región.

De todo lo anterior se puede inferir que los resultados obtenidos para este grupo de instituciones, a excepción de la institución 1 (INT-1, con una tasa de 0.54$)$, no están en correspondencia con las potencialidades de acuerdo con la regla de decisión establecida para la interpretación del resultado de este Número Índice, lo cual puede estar indicando un desacelerado ritmo del desarrollo o crecimiento disciplinar entre este grupo de instituciones; quizás debido a que el perfil de la mayoría de las instituciones participantes en este estudio es docente y sólo una tiene como actividad principal la investigación y la producción de nuevo conocimiento, ello no obstante que los docentes tienen contemplado dentro de su plan de trabajo la participación en proyectos de investigación y la publicación de sus resultados, y que los categorizados como investigadores participan también activamente en la impartición de docencia.

\section{Discusión}

Los resultados anteriores centran el debate en dos aspectos fundamentales: en primer lugar, que no siempre las instituciones académicas con mayores niveles de producción científica, sustentada en grandes concentraciones de potencialidades y recursos humanos y materiales, mantienen un ritmo de crecimiento 
con tendencia al desarrollo, y en segundo lugar, que instituciones con bajos niveles tanto de producción como de potencial científico pueden alcanzar una mejor tendencia al desarrollo de la disciplina, por la forma óptima con la que aprovechan sus potencialidades, debido a que poseen mayores niveles de productividad.

Por otra parte pareciera que las potencialidades mantienen una relación directamente proporcional con la producción científica; es decir, que a mayores potencialidades mayores niveles de producción científica, como es el caso de cuatro de las cinco instituciones estudiadas en este trabajo, tal y como se ilustra en el gráfico de la Figura 6.

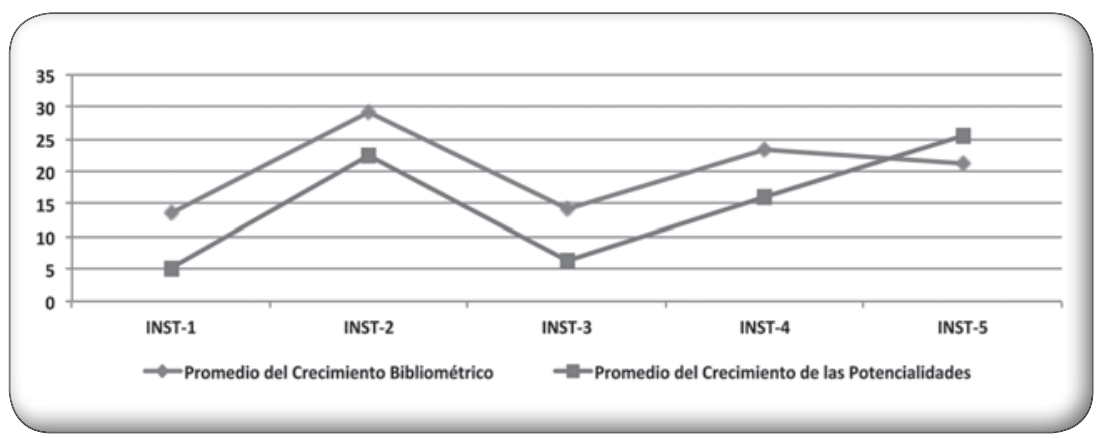

Figura 6. Distribución de instituciones según el valor promedio de crecimiento en indicadores bibliométricos y de potencialidades

Cuatro de las instituciones estudiadas mantienen similar ritmo de crecimiento en los dos sistemas de indicadores (los bibliométricos y los de potencialidades), a diferencia de la institución 5 que presenta una caída en el crecimiento de los indicadores bibliométricos, aspecto que indica que sus potencialidades se encuentran por encima de su producción. Este bajo aprovechamiento en sus recursos la sitúa en el último lugar de los valores obtenidos con el Índice de Desarrollo Disciplinar.

En este sentido el gráfico de la Figura 7 demuestra cómo el cálculo del valor promedio total por institución, obtenido entre ambos niveles de crecimiento (bibliométrico y de las potencialidades), relacionado con los valores obtenidos con el indicador propuesto, corrobora el supuesto que se ha venido comprobando durante todo este artículo, basado en el hecho de que el nivel acelerado de desarrollo disciplinar en una institución es directamente proporcional a la optimización que dicha institución hace con las potencialidades de investigación y docencia de las que dispone para generar sus productos científicos. 
En la Figura 7 se observa cómo la primera institución (INST-1) obtiene el primer lugar con el cálculo del indicador propuesto, no obstante que presenta el menor ritmo de crecimiento en ambos sistemas de indicadores.

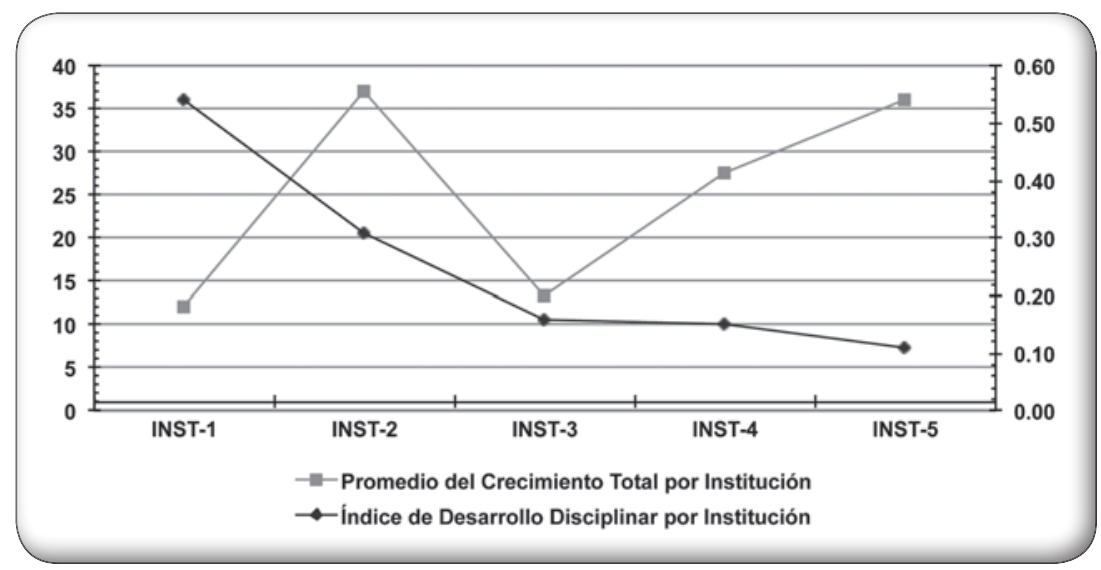

Figura 7. Distribución de instituciones según el valor promedio del crecimiento total y el nivel de Desarrollo Disciplinar alcanzado con el Idd

Coincidentemente los valores obtenidos por cada institución calculados con el Idd decrecen en función de su orden numérico, independientemente del ritmo de crecimiento global que hayan alcanzado en los dos sistemas de indicadores, con lo que demuestran que la optimización de las potencialidades y los altos niveles de crecimiento en estos dos tipos de indicadores (bibliométricos y los de potencialidades) no siempre están asociados con el desarrollo disciplinar de la institución, sino más bien que el crecimiento desproporcionado de ambos indicadores podría llegar a un fenómeno de saturación y estancamiento.

\section{CONSIDERACIONES FINALES}

Los resultados finales obtenidos con el cálculo del indicador propuesto demuestran que el desarrollo disciplinar alcanzado por cada institución es diferente, y que éste no está determinado por el volumen de las potencialidades que poseen ni por la producción y comunicación científica que generan, sino más bien por el aprovechamiento que hacen de las primeras y por la forma con la que producen las segundas.

Resulta oportuno considerar que los resultados obtenidos con este indicador no deben ser interpretados como una medida de la calidad del desempeño 
académico de las instituciones o de sus productos, puesto que en sus variables no figuran (ni sería conveniente su inclusión a posteriori) aquellos aspectos que se relacionen con la calidad, debido a la complejidad de las variables que se orientan a estos fines y el componente subjetivo o cualitativo que está presente en los aspectos relacionados con la calidad. Sin embargo este indicador sí puede ser considerado como una medida que identifica la tendencia de desarrollo de un conjunto de instituciones y, por consiguiente, de una disciplina en determinado país o región, sustentada en el nivel de aprovechamiento que la institución hace de sus potencialidades y no en el volumen de los recursos del que dispone, por lo que este indicador pudiera ser considerado también como un índice de la eficacia técnica de la institución en el aprovechamiento de su potencial académico, lo cual puede estar revelando una mayor tendencia hacia el desarrollo científico institucional y disciplinar.

De acuerdo con los valores asumidos para la interpretación de los resultados obtenidos con la aplicación del Índice de Desarrollo Disciplinar en este grupo de instituciones, se pudo comprobar que sólo una institución (INST-1) presenta mejor nivel de desarrollo en la disciplina, debido a que su valor (0.54) se encuentra, aunque con muy poco margen, por encima del 0.5, no obstante que en el cálculo del crecimiento esta institución es la que menor nivel posee en su proyección para el año 2012.

La metodología y el indicador propuesto para la medición del desarrollo disciplinar en este trabajo, así como los resultados obtenidos en la medición de las cinco instituciones, demuestran la factibilidad de su uso no sólo en el campo de las Ciencias Bibliotecológica y de la Información, sino que también pueden ser aplicadas a cualquier otro campo de conocimiento en países de economías periféricas o en desarrollo.

\section{Agradecimiento:}

Los autores desean expresar su más sincera gratitud al Profesor Juan José Piña Pozas por sus observaciones y comentarios relacionados con la formulación del Número Índice, así como a las sugerencias y recomendaciones aportadas por los árbitros, todas en beneficio y enriquecimiento de este artículo. 


\section{REFERENCIAS}

Caves, D.; Christensen, L. y Diewert, W. (1982), "The Economic theory of Index Numbers and the Measurement of Input, Output and Productivity”, en Econométrica, 50(6).

Cozzens, S. E. (1990), Literature-Based data in research evaluation: a manager's guide to bibliometrics, SPSG Concept Paper núm. 11. London: Science Policy Support Group, 25 pp.

Gómez-Caridad, I. y Bordons-Gangas, M. (1996), "Limitaciones en el uso de los indicadores bibliométricos para la evaluación científica”, en Política Cientifica, 46, pp. 21-26.

Gorbea-Portal, S. (2010), "Potencialidades de investigación y docencia Iberoamericanas en Ciencia Bibliotecológica y de la Información”, en Potencialidades de investigación y docencia Iberoamericanas en Ciencias Bibliotecológica y de la Información. Memoria, México: UNAM, Centro Universitario de Investigaciones Bibliotecológicas, pp. 3-38.

López-Piñero, J. M. y Terrada, M. L. (1992), "Los indicadores bibliométricos y la evaluación de la actividad médico-científica (IV). La aplicación de los indicadores”, en Medicina Clínica (Barc.), Barcelona: Doyma, vol. 98, c1992, pp. 384-388.

Maltrás-Barba, B. (2003), Los indicadores bibliométricos: fundamentos y aplicación al análisis de la ciencia, Asturias, España: TREA, 272 pp.

Mason, R. D. y Douglas, A. L. (1992), Estadística para administración y economía, México: Alfaomega.

Narin, F. (1976), Evaluative Bibliometrics: The use of publication and citation analysis in the evaluation of scientific activity, Cherry Hill, New Jersey: Computer Horizons, Inc., 459 pp.

OCDE (1993), "The measurement of scientific and technological activities. Proposed standard practice for surveys of research and experimental development", en Frascati Manual 1993, París: OCDE.

- - - (1995),"The measurement of scientific and technological activities: manual on the measurement of human resources devoted to S\&T", en Camberra Manual, París.

Piña-Pozas, M. M. (2011), Modelo de indicadores cienciométricos y bibliométricos para la interpretación del desarrollo de las Ciencias Bibliotecológica y de la Información en México, Tesis doctoral, Universidad Nacional Autónoma de México, México.

Ribeiro, W.; Rodríguez, E. y Cervini, M. (2008), “Capítulo III. Números Índice: conceptos y aspectos generales”, en Economía, Uruguay: Universidad de la República.

Russell Barnard, J. M. (2004), “Obtención de indicadores bibliométricos a partir de la utilización de las herramientas tradicionales de información", en Congreso internacional de la información INFO 2004, Ciudad de La Habana, 12 al 16 de abril. 
Sánchez Fernández, J. (2004), “Capítulo 5. Números Índices”, en Introducción a la Estadística Empresarial, España: EUMEDNET.

Sancho, R. (1990), "Indicadores bibliométricos utilizados en la evaluación de la ciencia y la tecnología”, Revisión bibliográfica, en Revista Española de Documentación Cientifica, vol. 13, núm. 3-4, pp. 842-865.

- - - (1996), "Manual de Frascati para la medición de las actividades científicas y técnicas", en Política Científica, vol. 45, marzo, pp. 21-26.

Squires, D. (1988), Index numbers and productivity measurement in multispecies Fisheries: an application to the pacific coast trawl fleet, U.S. Department of Commerce.

Suárez Balseiro, C. A. (2004), Modelo para la evaluación de la actividad investigadora en el ámbito universitario: el caso de la Universidad Carlos III de Madrid, Tesis doctoral, Universidad Carlos III de Madrid, Madrid, España.

Torres-Salinas, D. y Jiménez-Contreras, E. (2012), "Hacia las unidades de bibliometría en las universidades: Modelo y funciones", en Revista Española de Documentación Cientifica, vol. 35, núm. 3, julio-septiembre.

Van Raan, A. F. J. (1996), "Advanced bibliometric methods as quantitative core of peer review based evaluation and foresight exercises”, en Scientometrics, vol. 36, núm. 3, pp. 397-420. 


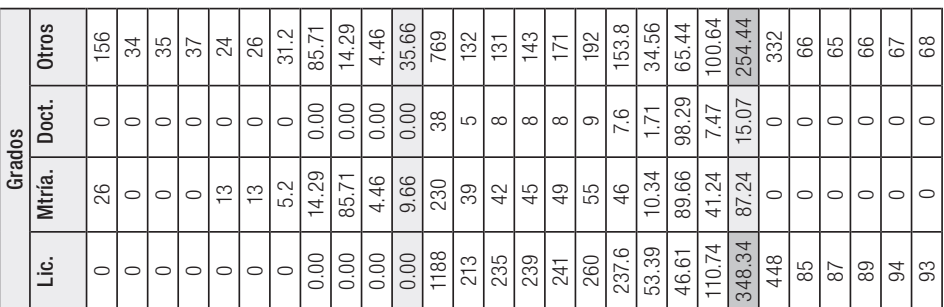

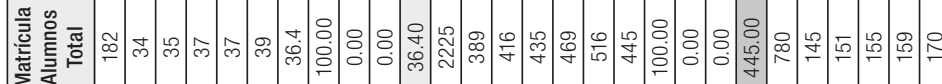

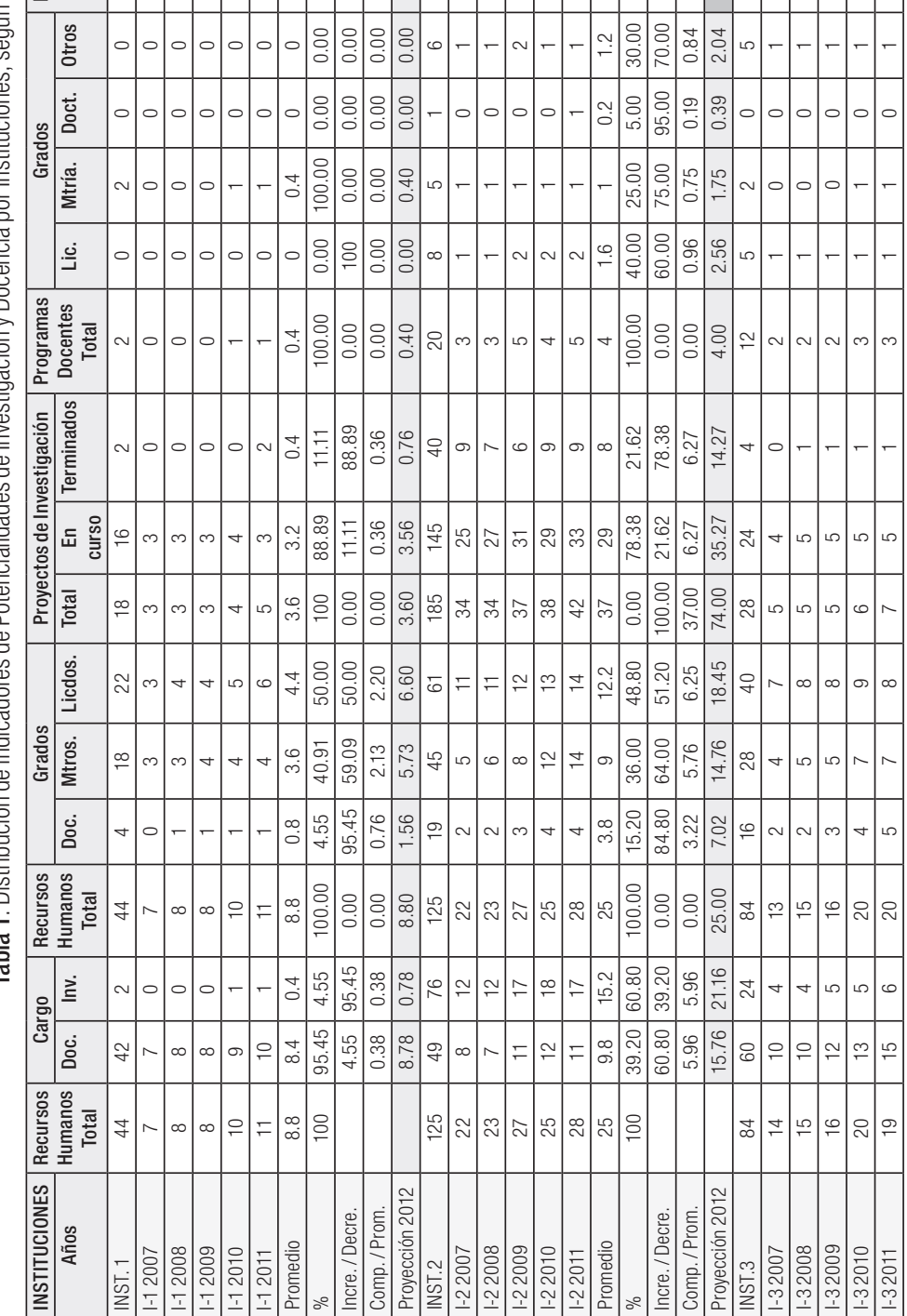




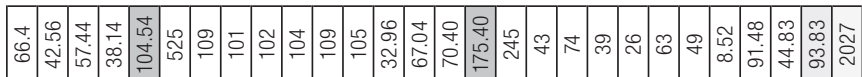

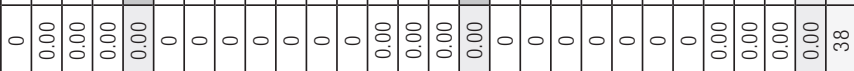

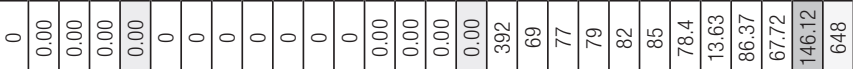

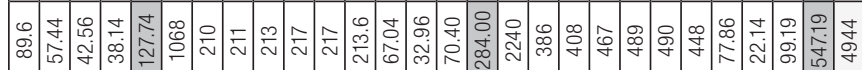

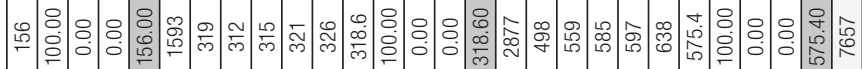

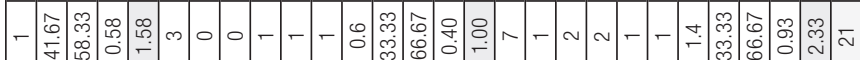

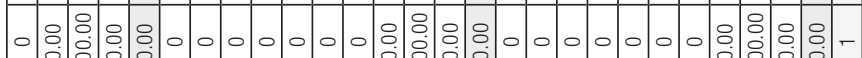

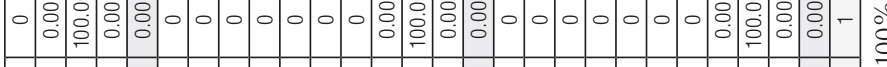

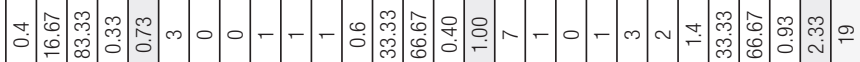

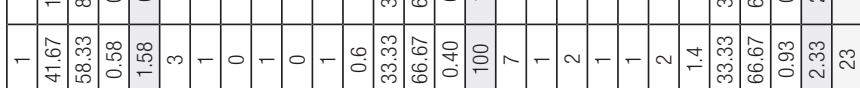

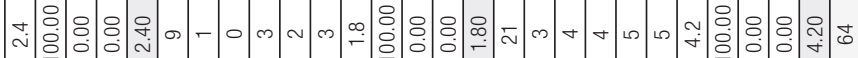

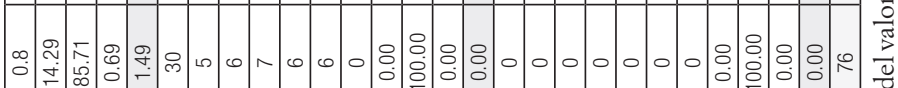

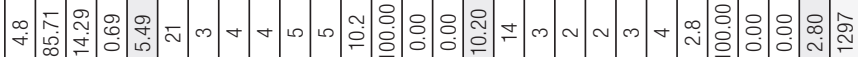

ம.

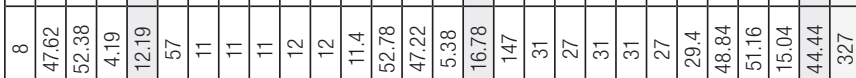
0. ம் ^ง

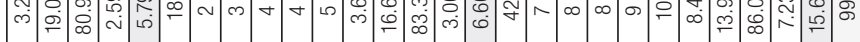
皮定 $=\frac{0}{\pi}$ 范 $\therefore$ 至

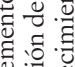
: 인 ำ 过

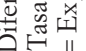

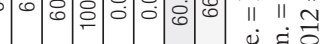

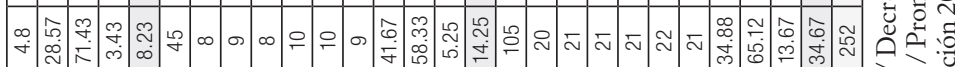

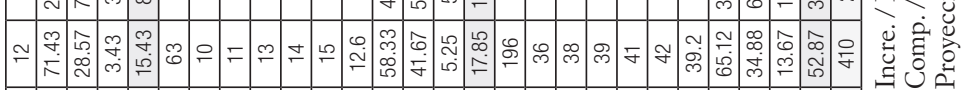

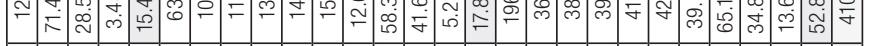

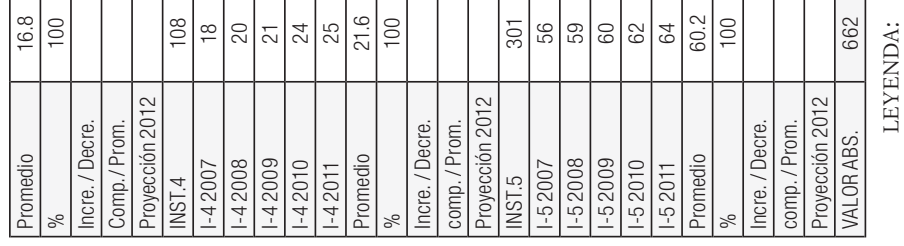




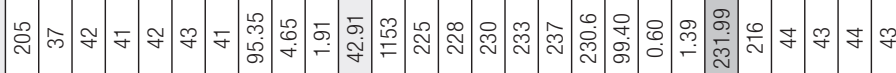

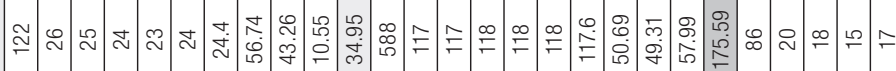
음

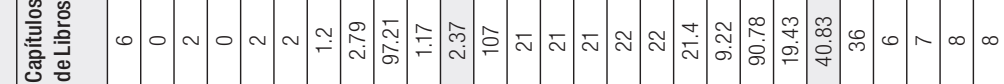

:

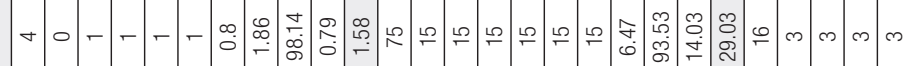

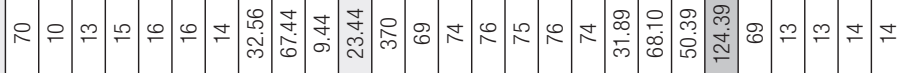

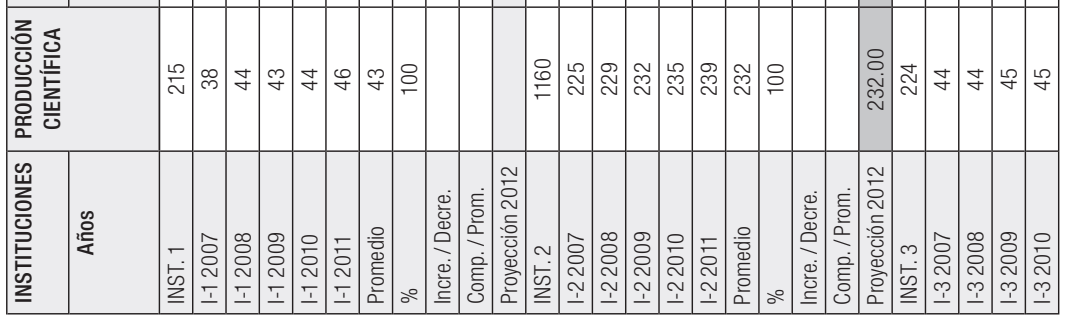




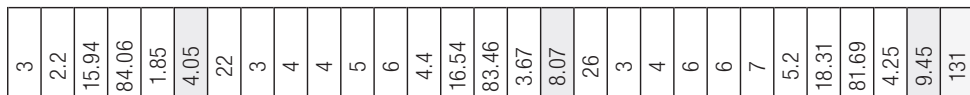

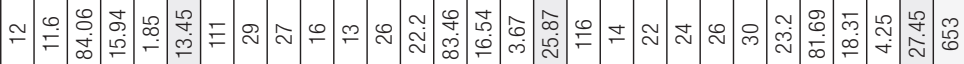

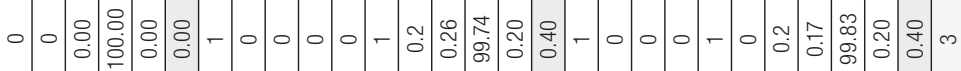

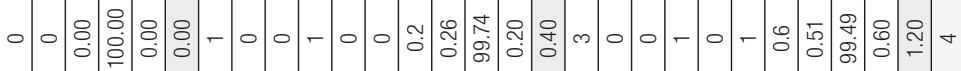

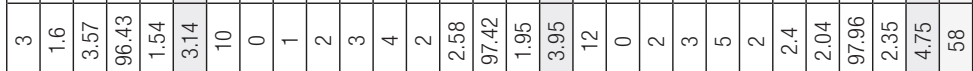

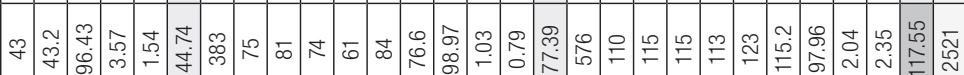

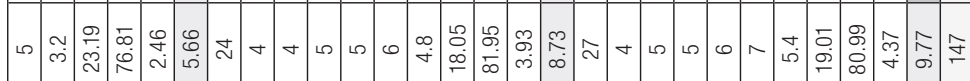

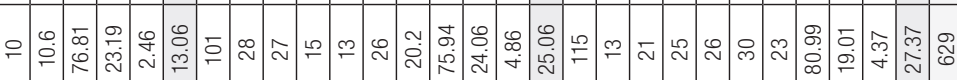

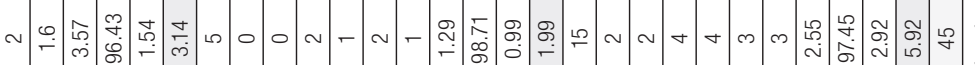

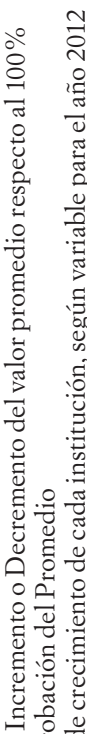

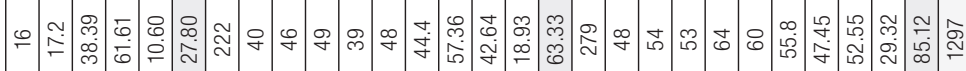

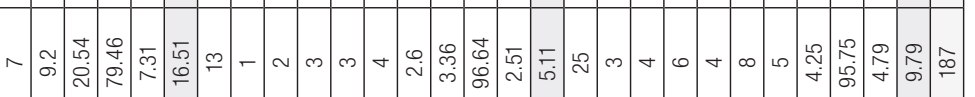

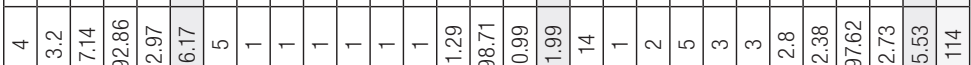

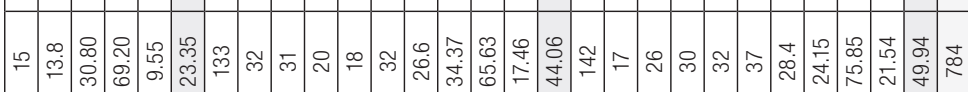
造

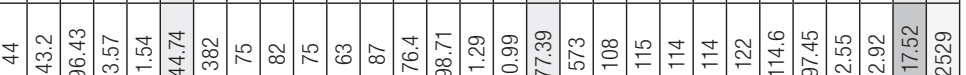

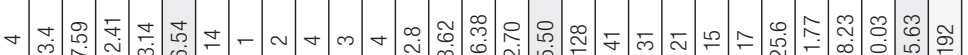

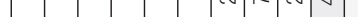
ن $\dot{\text { है }}$

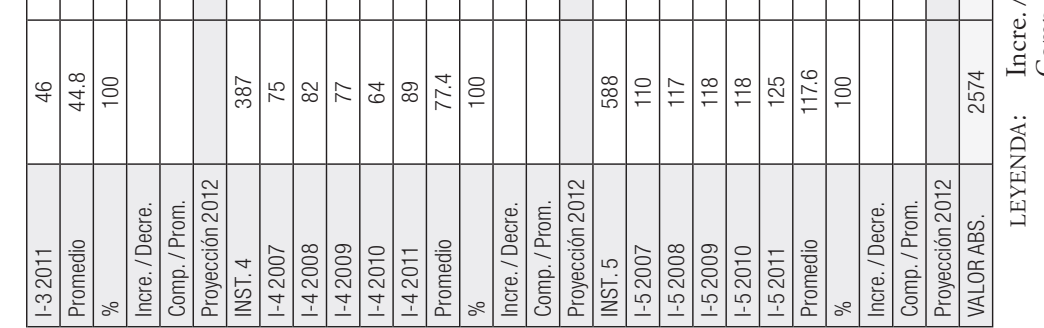
它流 

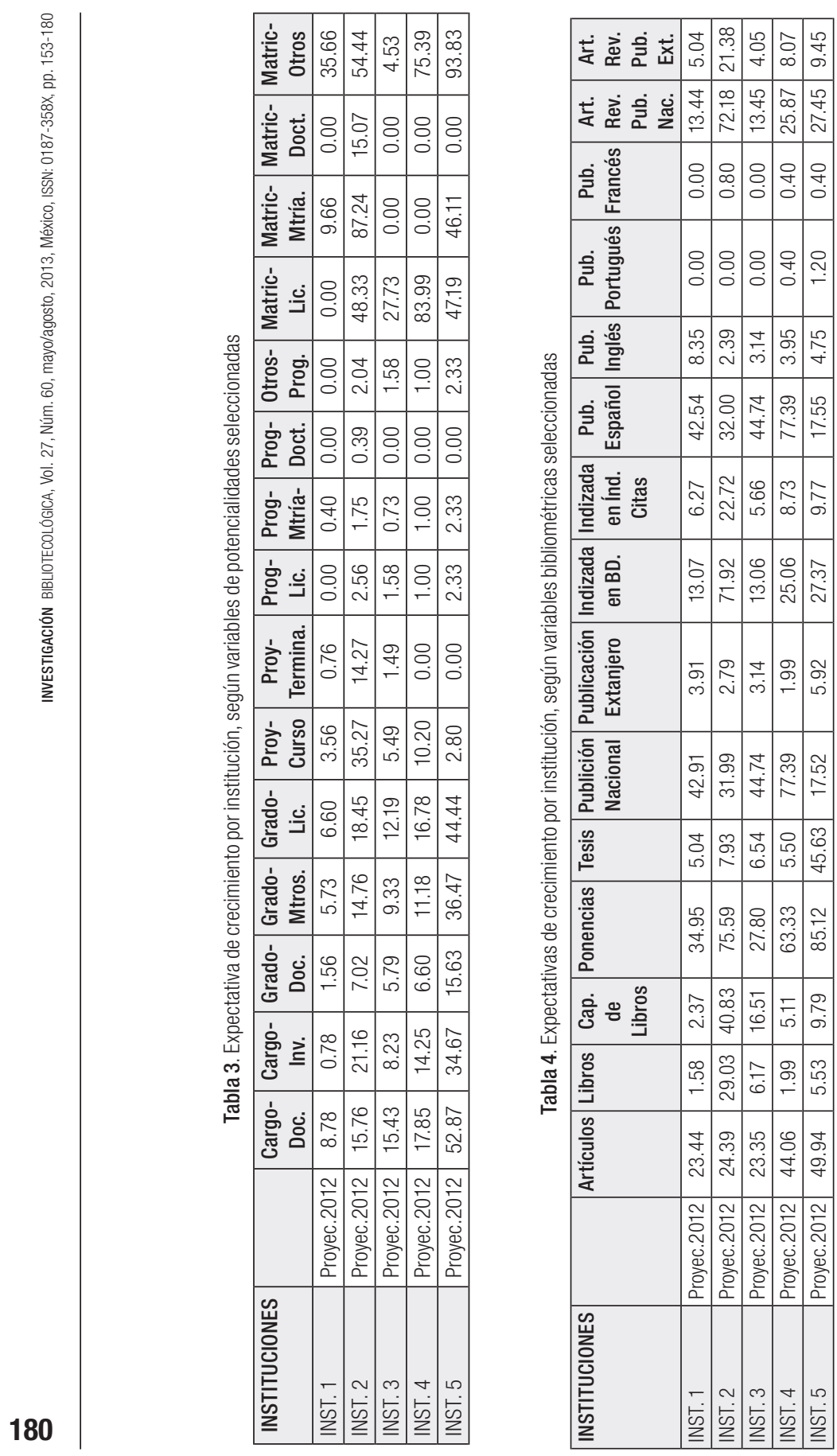